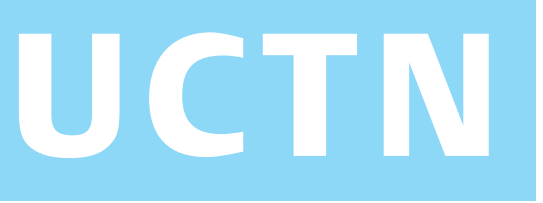

\title{
A New Approach to the Bile Duct via Needle Puncture of the Papillary Roof
}

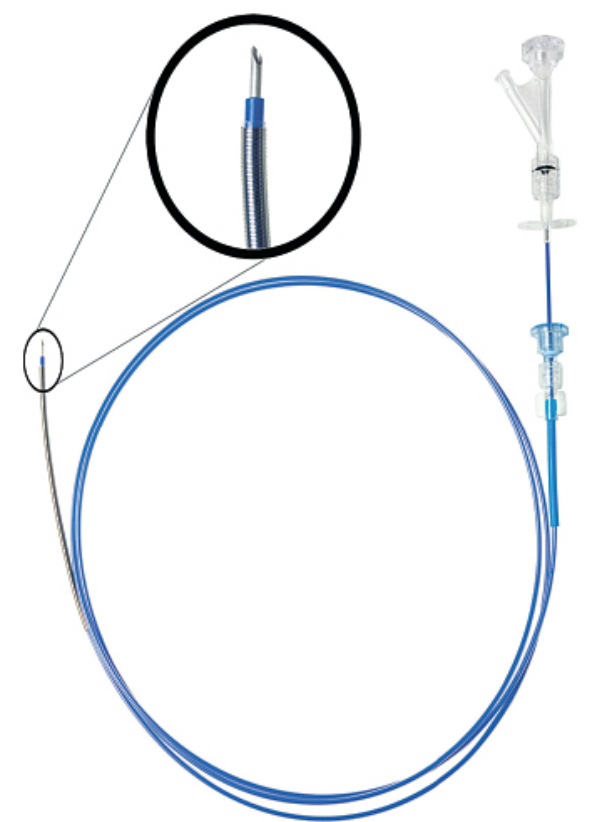

Figure 1 The CMS-Medical catheter with an 18-gauge needle, covered by a flexible metallic sheath at the distal end.

Precut is an alternative technique for bile duct access when the conventional cannulation method has failed, although it is considered to be associated with a high risk of complication by acute pancreatitis $[1,2]$.

We describe an approach to the bile duct via puncture of the papillary roof without endoscopic ultrasound control, using a new design of catheter (CMS-Medical, São Paulo, Brazil). This catheter is made of polyethylene, with an 18-gauge needle covered by a flexible metallic sheath at the distal end (Figure 1). The puncture is made in the midline of the proximal roof of the papilla, along the axis of the bile duct. After puncturing, a 0.025/0.018inch guide wire is gently pushed through the catheter and into the bile duct under fluoroscopic control. A double-lumen catheter for cholangiography is then slid over the wire into the bile duct and the contrast medium is injected (Figure 2 ). If there is resistance to progression of the guide wire, a fresh attempt at puncture of the papilla should be made in order to access the bile duct.

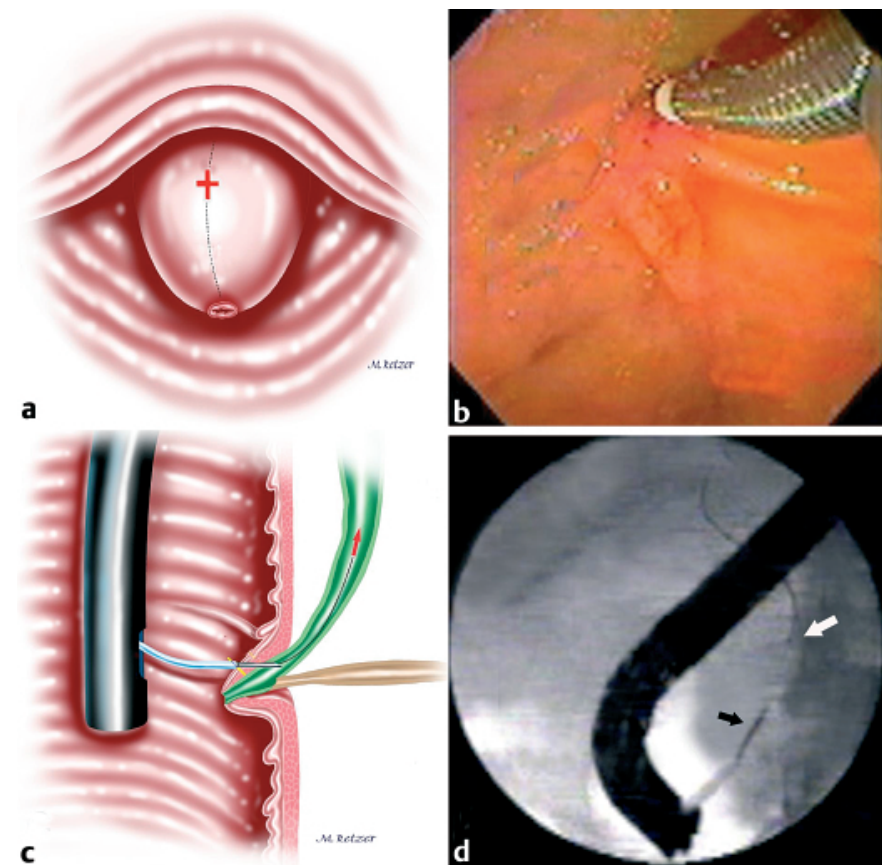

Figure 2 Schematic view (a) and endoscopic view (b) of the puncture site in the roof of the papilla. c Placement of the guide wire through the catheter into the common bile duct. d Radiological view showing the needle (black arrow) and the guide wire (white arrow).

This new technique represents a safe, low-cost approach to gaining access to the bile duct for investigative and therapeutic procedures. It is not complicated by thermal injury or by the acute pancreatitis associated with sphincterotomy [3].

\section{E. L. A. Artifon, F. Y. Hondo, P. Sakai, S. Ishioka}

Gastrointestinal Endoscopy Unit, São Paulo University Medical School, São Paulo, Brazil

\section{Reference}

${ }^{1}$ Masci E, Mariani A, Curioni S et al. Risk factors for pancreatitis following endoscopic retrograde cholangiopancreatography: a meta-analysis. Endoscopy 2003; 35: $830-834$

2 Schapira L, Khawaja FI. Endoscopic fistulo-sphincterotomy: an alternative method of sphincterotomy using a new sphincterotome. Endoscopy 1982; 14 : $58-60$
${ }^{3}$ Mavrogiannis C, Liatsos C, Romanos A et al. Needle-knife fistulotomy versus needle-knife precut papillotomy for the treatment of common bile duct stones. Gastrointest Endosc 1999; 50: 334- 339

\section{Corresponding Author}

\section{E. L. A. Artifon, M.D.}

Gastrointestinal Endoscopy Unit, São Paulo University Medical School, São Paulo, Brazil

R. Panamericana, 64

São Judas 04303-170

São Paulo SP

Brazil

Fax: $\quad+55-11-3083-5216$

E-mail: eartifon@yahoo.com 